\title{
Abdominal Tuberculosis - A Clinical Perplexity In Diagnosing Acute Abdomen: Case series
}

\author{
Dr. Harika Kapu ${ }^{1}$, Dr. R.K.Shastri ${ }^{2}$, Dr. Kagitha Usha Rani ${ }^{3}$ \\ 1,3 ( Post Graduate in General Surgery, Dr.Pinnamaneni Siddhartha Institute Of Medical Sciences \& Research \\ Centre,chinaoutapalli, Krishna Dist, Andhra Pradesh, India.) \\ 2 ( Professor Of General Surgery, Dr.Pinnamaneni Siddhartha Institute Of Medical Sciences \& Research \\ Centre, chinaoutapalli, Krishna Dist, Andhra Pradesh, India ).
}

\begin{abstract}
Abdominal tuberculosis accounts for about 10\% among the acute abdomen cases. The diagnosis of acute abdomen cases is confusing, especially when there is no history of primary pulmonary tuberculosis. It is a diagnostic challenge to differentiate it from other diseases such as infectious processes, tumors, peri appendiceal abscesses and Crohn's disease. We report a case series of acute abdomen cases which created a perplexity in our diagnosis and there arises a necessity to remember about tuberculosis as a probability in acute abdomen cases. Irrespective of surgery, all patients of abdominal tuberculosis require a full ATT [Anti Tuberculosis Treatment]course.

Keywords: Acute abdomen, Abdominal Tuberculosis, Acute Appendicitis, Intestinal Obstruction
\end{abstract}

\section{Introduction}

Abdominal tuberculosis includes tuberculosis infection of Gastro Intestinal tract, mesentery, lymph nodes and omentum, the peritoneum and related solid organs such as liver and spleen ${ }^{(1,2)}$.The initial clinical presentations are nonspecific as the disease involves multiple sites with different morphology. No single lab investigation is pathagnomic ${ }^{(1)}$. Radiology often fails to reveal the classic changes described in surgical text books. Bacterial culture and tissue histopathology though confirmatory are time consuming and immunological tests though rewarding are expensive. Moreover, abdominal tuberculosis with an acute abdomen presents as an enormous challenge to surgeon. A surgeon has to rely on his clinical judgment \& surgical assumes to determine the extent of surgical management in an unprepared, physiologically, compromised patient in the emergency setting. While providing surgical care, surgeons have to collect sufficient pathological tissues for histopathology to overcome the diagnostic perplexity ${ }^{(1,2)}$.

We are reporting the 3 cases, which were presented as acute abdomen diagnosed as different acute abdominal conditions but came out to be Abdominal Tuberculosis.

\section{Case 1}

A Male patient aged 28 yrs came with a chief complaint of pain abdomen from past 6 days along with fever with chills and rigors associated with vomitings. Similar episodes from past 6 months i.e. 7 episodes of pain abdomen with vomitings containing food particles. On per abdomen examination, tenderness was present in right iliac fossa. A mass of $7 * 5 \mathrm{~cm}$ present in right iliac fossa oval in shape firm consistency with all borders well defined.

Ultrasound abdomen \& pelvis showed Ileo-ceacal junction appears thickened and heterogeneous with surrounding mesenteric inflammation with free fluid in right iliac fossa these features are in suggestive of acute appendicitis with possible mass formation. Interval appendicectomy was planned. But patient was not relieved from symptoms and mass was not reduced and plan was changed to exploratory laparotomy.

Intra-operative findings are Ileo-ceacal lump with multiple tubercles studded on the surface of lump and terminal ileum and jejunum [fig1] and partial strictures were found in jejunum [fig2]. Multiple mesenteric lymph nodes were present. Serosa along with mesenteric lymph nodes were sent for biopsy. Biopsy Report showed Chronic Granulomatous Lesion suggesting of Koch's Etiology and patient was started on ATT[ Anti Tuberculosis Treatment]. 


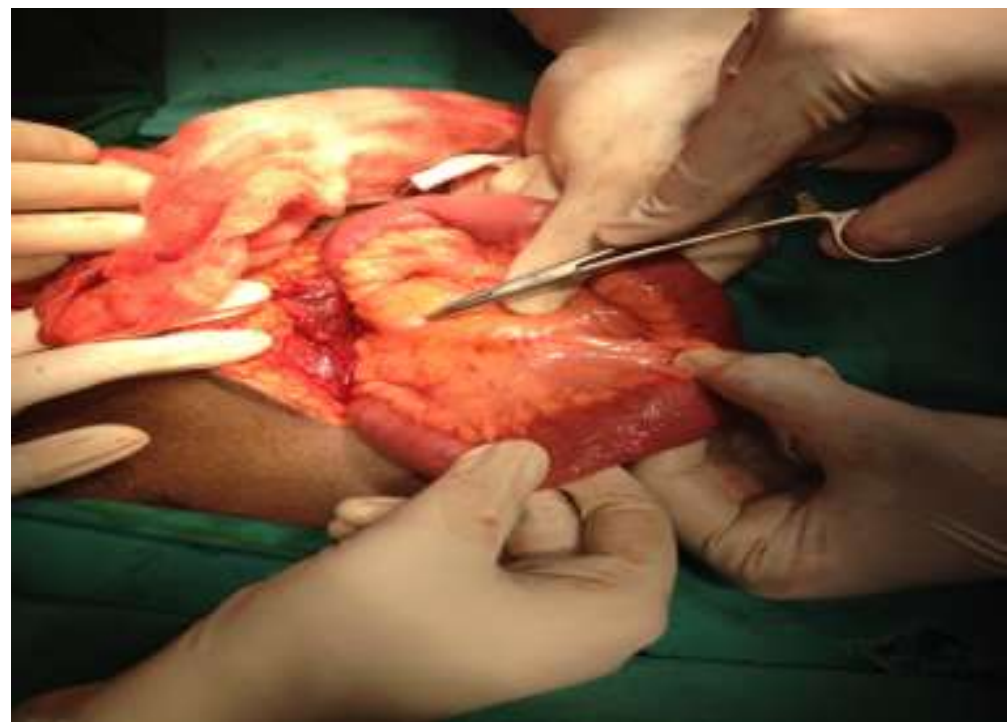

Fig1

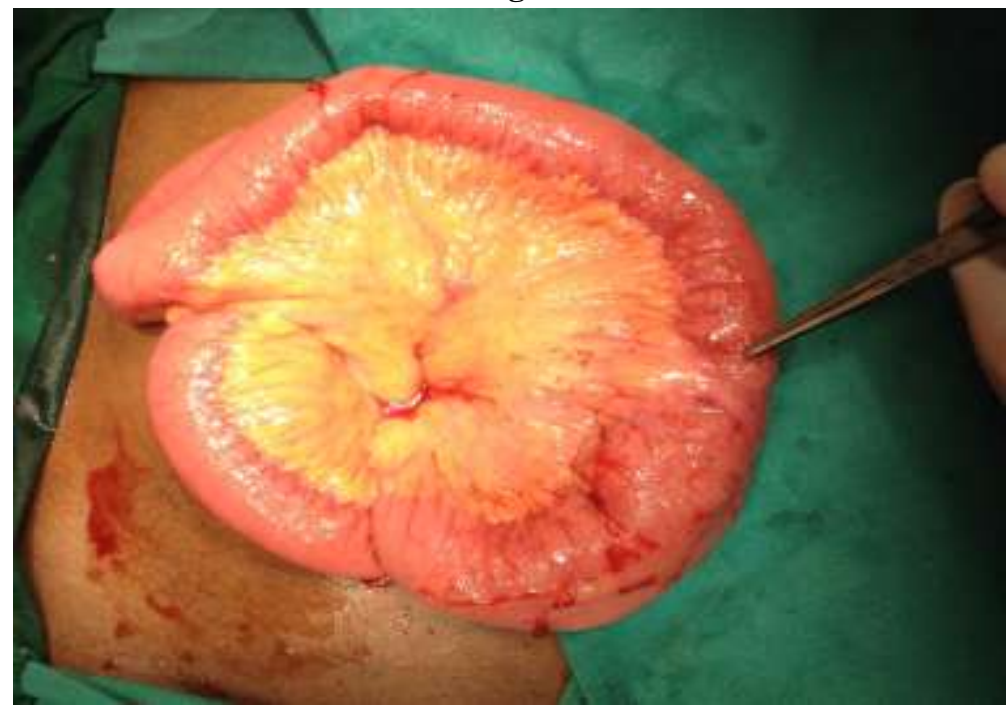

Fig2

II. Case 2

A female patient aged 22yr came with chief complaint of pain abdomen and vomiting for 1 week and with a history of enteric fever 3 months back. Diagnostic lap was done 20 days back. Ascitic fluid was taken and sent for AFB and PCR both were negative. History of bronchial asthma was present and used medication only when symptomatic. No history of pulmonary tuberculosis. Patient was cachexic. On per abdomen examination, diffuse tenderness present all over the abdomen. Abdomen was soft with no guarding and rigidity and no palpable mass.

Ultrasound abdomen showed focal wall thickening involving ascending colon with Peripancreatic, paraaortic \& mesenteric lymphadenopathy. CECT abdomen and pelvis showed, Extensive necrotic lymphadenopathy in the peripancreatic region, mesentery and retro peritoneum .Wall thickening in the caecum, ascending colon with adjacent pericolic fat stranding and lymphadenopathy. Dilatation of small bowel loops with abnormal air fluid levels with abrupt narrowing in the ileum these features are in favor of SUBACUTE SMALL BOWEL OBSTRUCTION due to pathology in ileum. 


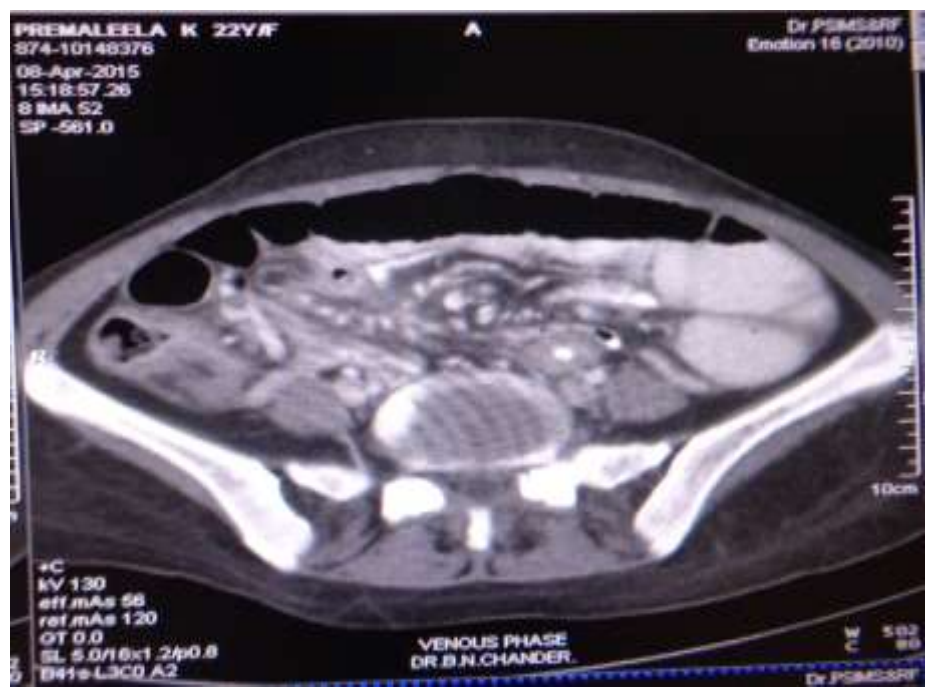

Fig 3

Intra operative findings are big hard lymph nodal mass $8 * 8 \mathrm{~cm}$ found in mesentery [fig4]. Multiple strictures (about 8 strictures) found at different sites from dudenojejunal junction to ileoceacal junction [fig5]. Segment of intestine with 2 strictures with impending obstruction were resected and anastomosis done [Fig 6] and was sent for biopsy along with lymph nodes. Biopsy came as chronic granulomatous lesion suggestive of Koch's etiology and patient was started on ATT [Anti Tuberculosis Treatment].

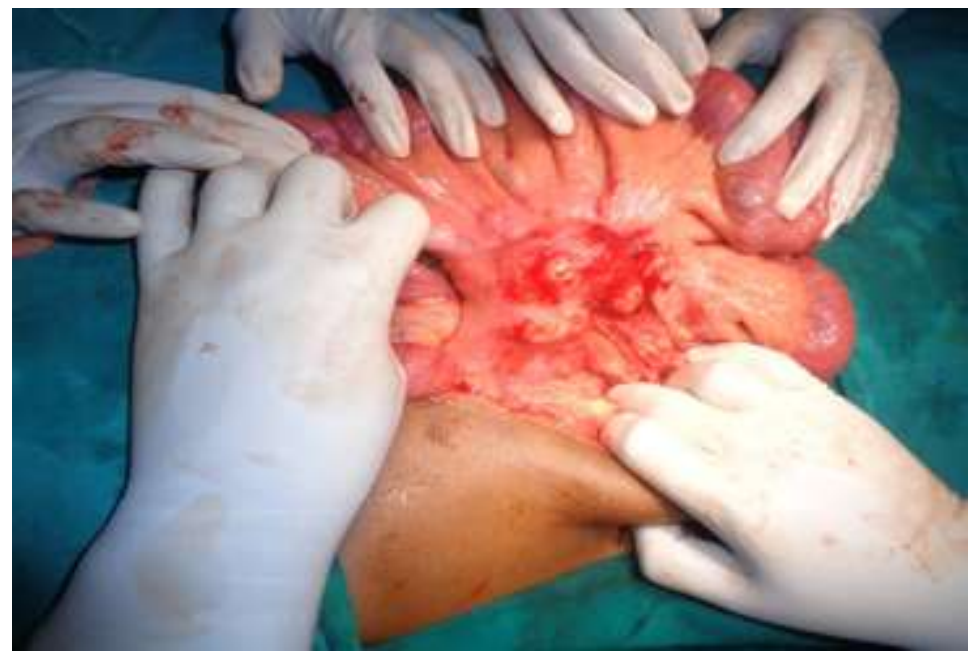

Fig4

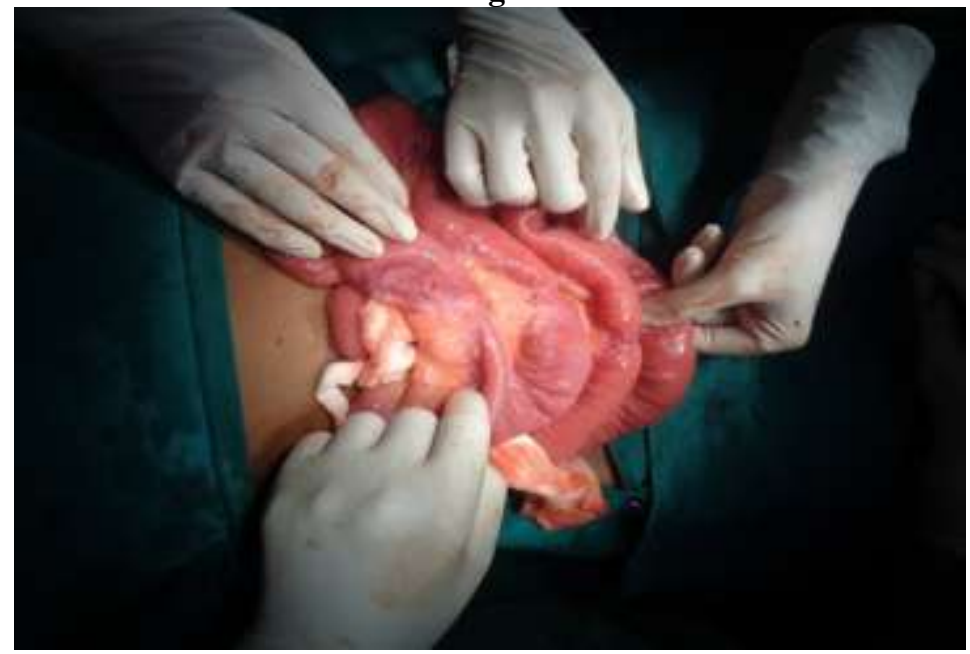

Fig-5 


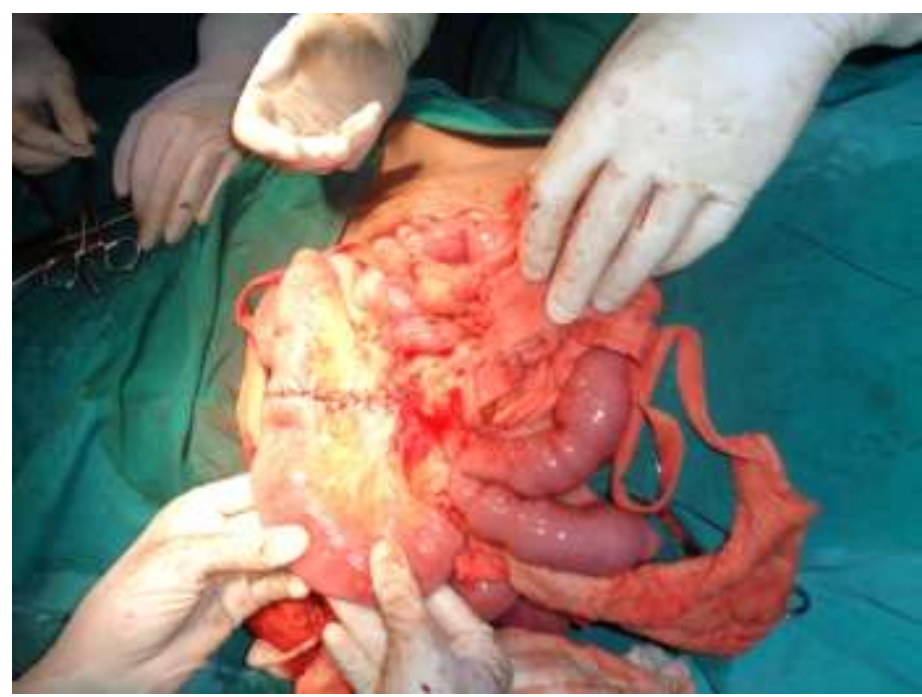

Fig 6

\section{Case 3}

A male patient aged 24yrs came with chief complaint of pain abdomen and vomitings for 20 days. Pain abdomen from 20 days which was colicky type lasting for 2-3 min with bilious vomitings which are fowl smelling, immediately after taking food 4-5 episodes per day .No history of fever. History of weight loss was present. Passing stools 2-3 episodes per day watery stools similar complaints in the past. Mother died with cancer, father died of pneumonia. On general examination, Patient was cachexic, alert, oriented. On per abdomen examination, tenderness present in right iliac fossa, no mass felt.

Ultrasound abdomen showed focal bowel wall thickening measuring $11 \mathrm{~mm}$ (possible sigmoid colon) is noted with possible fecolith at this point causing obstruction and proximal dilatation of bowel - to rule out INFECTIVE / NEOPLASTIC pathology. CECT abdomen [Fig 7,8] showed focal nodular wall thickening with severe luminal narrowing involving Distal jejunum/ Proximal ileal loops with features in favour of small bowel obstruction with mesenteric lymphadenopathy these features are in favour of Neoplastic etiology. Explorative Laparotomy was done.

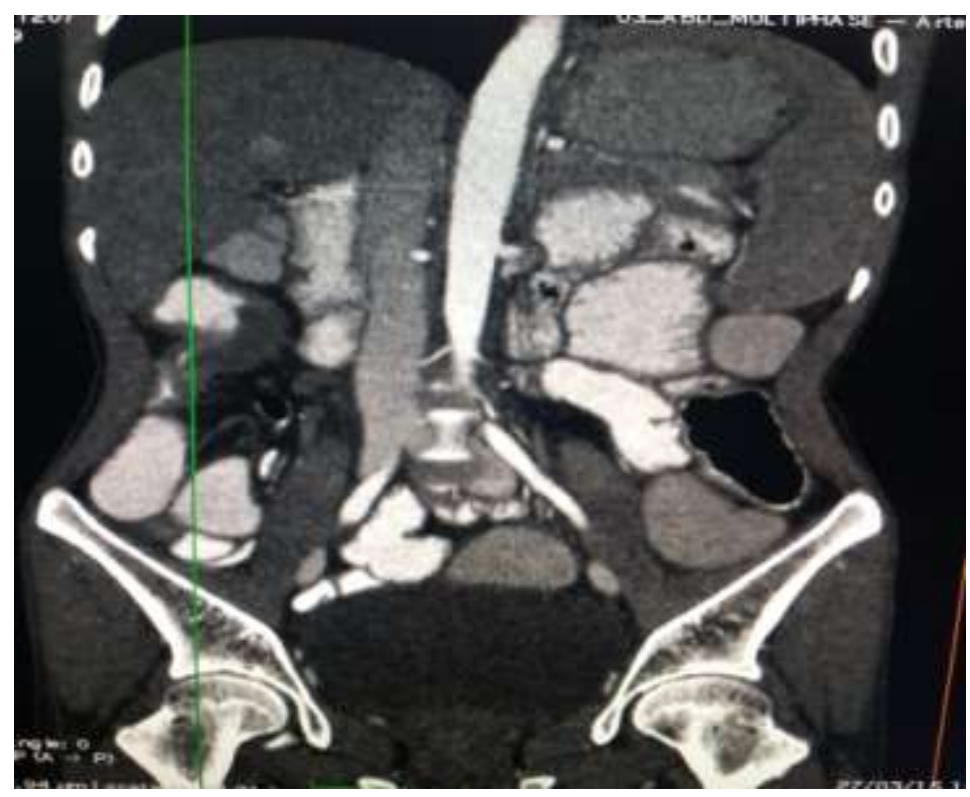

Fig 7 


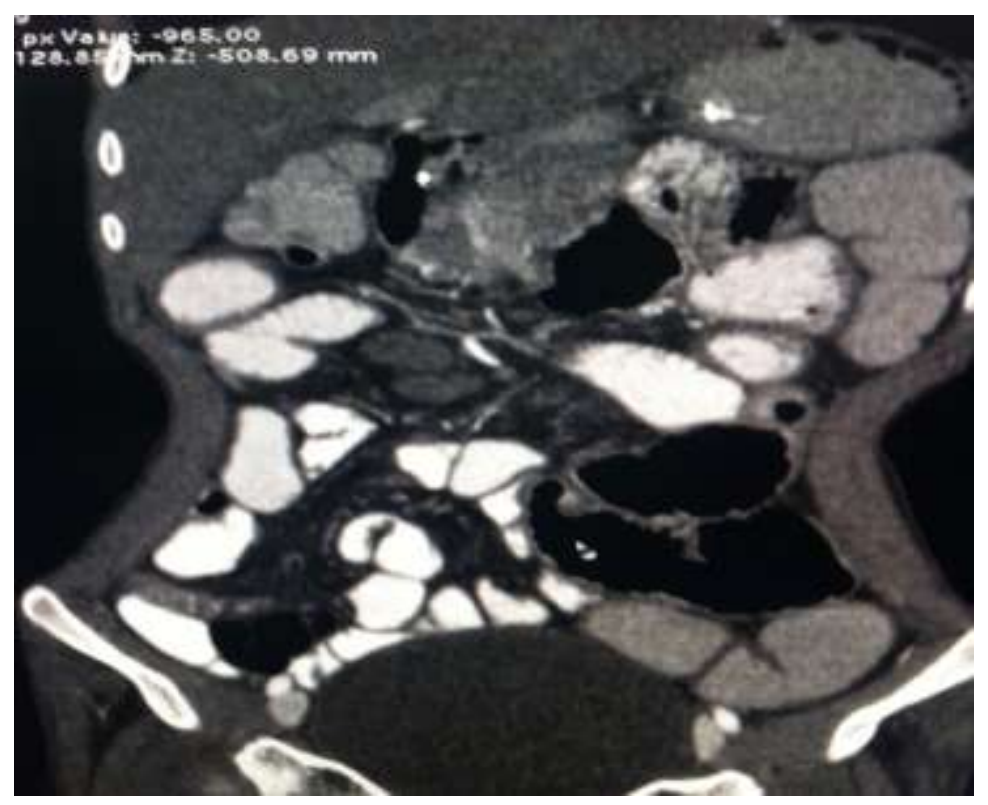

Fig 8

Intraoperative findings are dilated bowel loops proximal to the jejunal stricture $(3 \mathrm{cms})$ and collapsed bowel loops distal to it [fig9]. Mesenteric lymph node measuring $2 * 2 \mathrm{cms}$ identified near diseased segment, LN excised and sent for HPF [fig 10]. Structured disease jejunal segment is resected and end to end anastomosis was done [Fig 11, 12]. Biopsy report showed Chronic granulomatous lesion suggesting Koch's etiology and patient was started on ATT [Anti Tuberculosis Treatment].

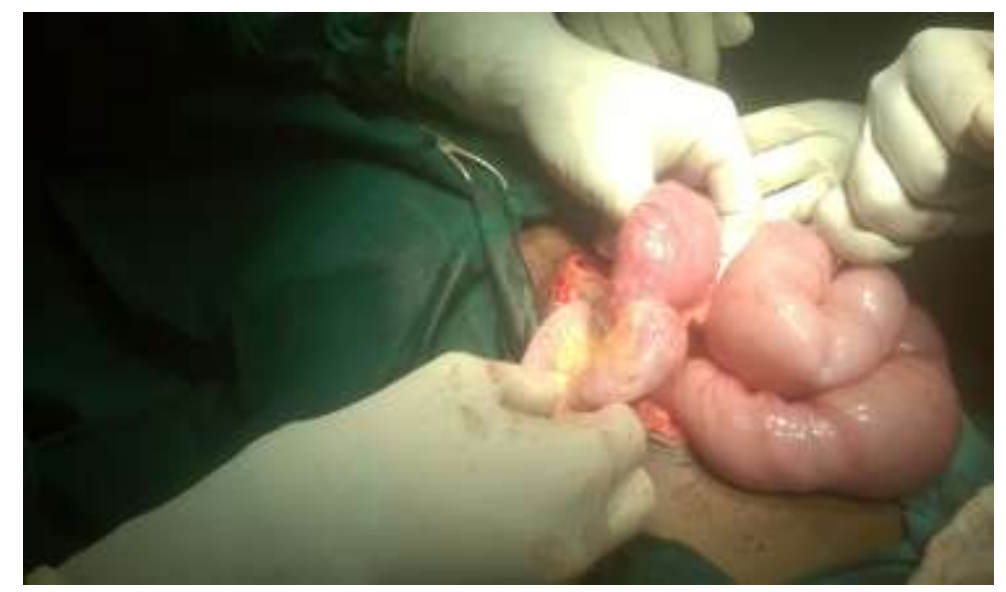

Fig 9

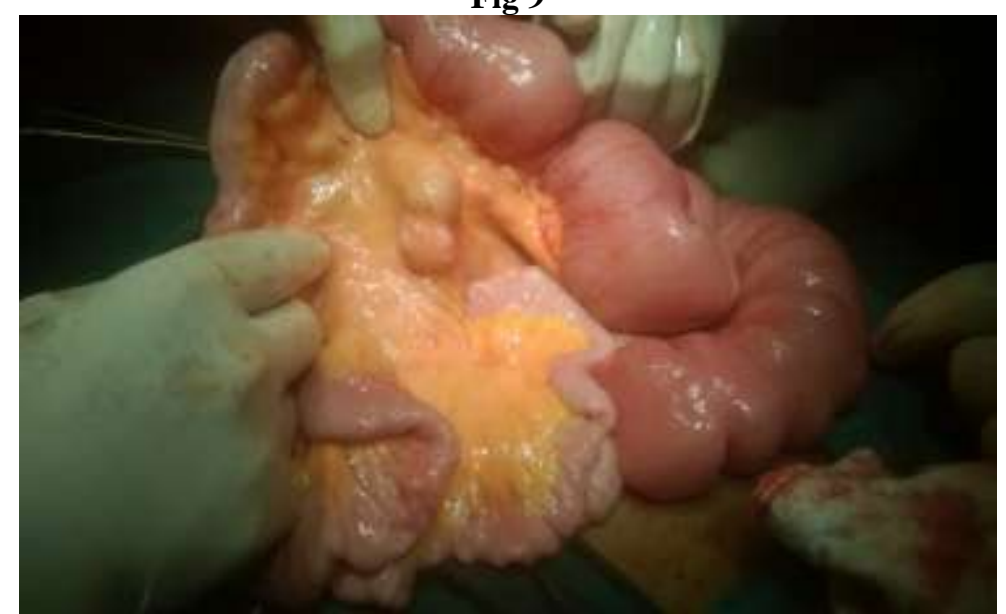

Fig 10 


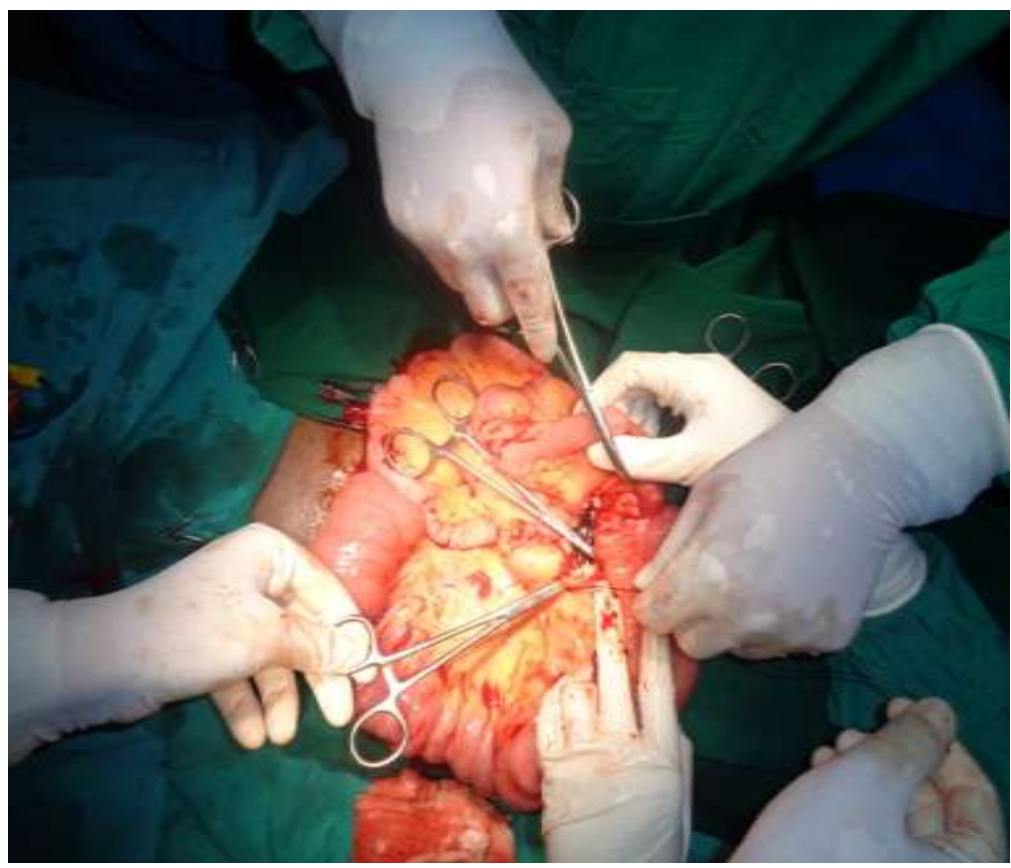

Fig 11

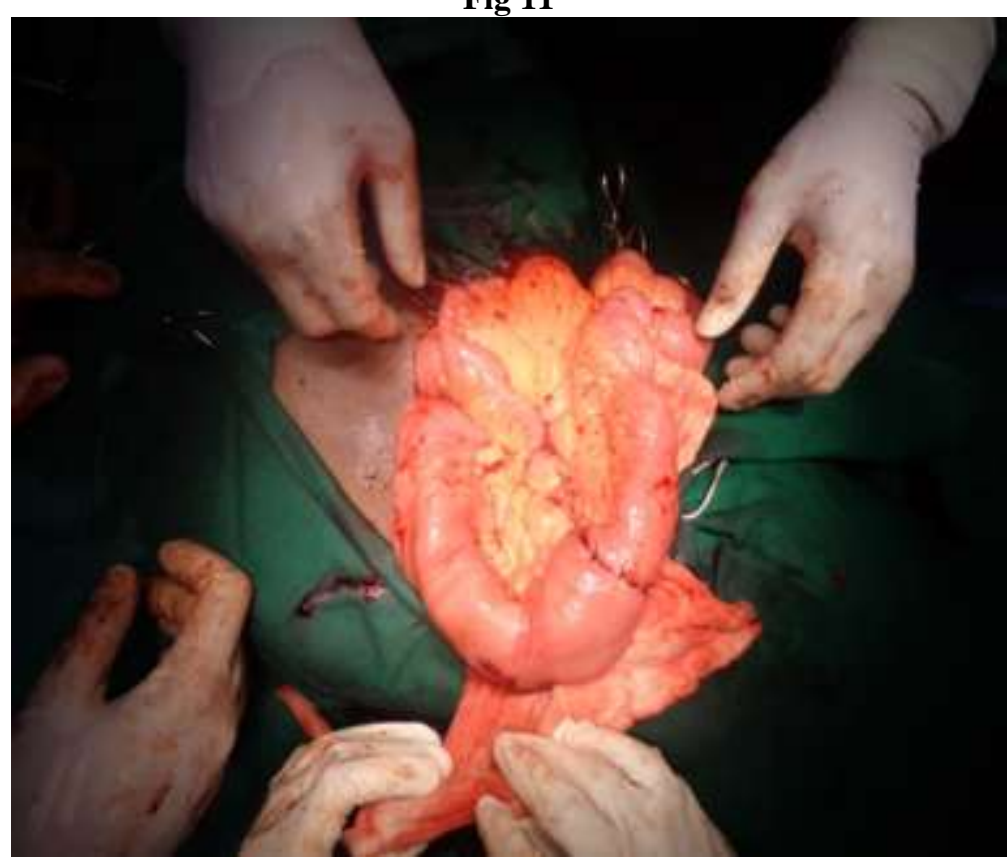

Fig12

\section{Discussion}

Abdominal tuberculosis contributes a major public health problem in developing countries and carries significant morbidity \& mortality ${ }^{(3,4,5)}$. It is predominantly seen in young age group ${ }^{(6)}$. The assumed routes of infection of GIT are ingestion, hematogenous spread from the lungs, from infected lymph nodes and direct spread from adjacent organs. Mostly caused by Mycobacterium tuberculosis seldom Mycobacterium.bovis from milk products. Manifestation of Gastro Intestinal tuberculosis are variable symptoms are nonspecific and include fever, night sweats, abdominal pain, weight loss and diarrhea, hemorrhage, obstruction, fistulization, perforation complications can occur which need surgical intervention. However, it remains considerable diagnostic challenge, especially in the absence of pulmonary infection, as it may mimic many other abdominal diseases such as infectious processes, tumors, peri- appendiceal abscesses and Crohn's disease $^{(8,9,10)}$. 
The colonoscopic features described in patients with colonic tuberculosis are transverse or linear ulcers, nodules, deformed ileo-ceacal valve and caecum and presence of inflammatory polyps. The main imaging techniques used are ultrasonography, CT, MRI. The common imaging features are enlarged para aortic nodes, asymmetric bowel wall thickening, ascites, inflammatory masses of the bowel wall lymph nodes and omentum , narrowing of the terminal ileum with thickening and gaping of ileo-ceacal valve, "white bowel" sign due to lymphatic infiltration and "sliced bread sign" due to fluid surrounding bowel caused by inflammation of bowel wall ${ }^{(7)}$.

The diagnostic procedure of choice would be colonoscopy and biopsy. We should depend upon histopathology, bacterial culture, and PCR for diagnosing abdominal tuberculosis. The differential diagnosis includes a broad spectrum of diseases. The clinical, radiological, and endoscopic picture is most likely to be confused with neoplasms or Crohn's disease and infrequently with other conditions including amoeboma, Yersinia infection, gastrointestinal histoplasmosis and peri- appendiceal abscess.

\section{Conclusion}

In all acute abdomen cases we should keep in our mind regarding abdominal tuberculosis as differential diagnosis. Abdominal tuberculosis presents as acute abdomen in late stages and always creates a perplexity in our diagnosis and we should remember about tuberculosis as a probability and depend on histopathological examination for early diagnosis and should be treated with ATT[Anti Tuberculosis Treatment]. In case of acute presentations surgical treatment would be choice for survival.

\section{References}

[1]. Kumar s, pandey HI, Saggu P, Abdominal tuberculosis in: Taylor 1and Johnson CD (Eds) Recent Advances of Surgery: 2008; 28:47-58.

[2]. Kapoor VK, Modern Management of Abdominal Tuberculosis, In: Taylor 1 and Johnson CD (Eds) Recent Advances of Surgery th 35 vol.2013: pp 156-69.

[3]. Butt J, Karamat KA, Ahmed RN, Mahmood A: Advances in diagnosis of Tuberculosis.Pak J Pathol. 2001,22:1-3.

[4]. Lonnroth K, Raviglion M: Global epidemiology of tuberculosis: Prospects for control semin Respiratory critical care Medicine 2008, 29:481.

[5]. Shaikh MS, Dholia KR, Jalbani MA: Prevelence of intestinal tuberculosis in cases of acute abdomen Pakistan J surg 2007, 23: 52-56.

[6]. Khan SM,Khan KM,Khan AS,Jehanzeb M,Jan WA,Khan M,Ali U:Presentation of abdominal tuberculosis in NWFP and its correlation with operative findings J Postgrad Med Inst 2005,19:286-291.

[7]. Donoghue HD, HoltonJ: Intestinal Tuberculosis Curr Opinn infects Dis 2009, 22:490-496.

[8]. Chatzicostas C,Koutroubakis IE,Tzardi M,Roussomoustakaki M,PrassopoulosP,Kouroumalis EA: Colonic tuberculosis mimicking Crohn's disease : case report .BMC Gastroenterol 2002,2:10.

[9]. Epstein D, Watermeyer G, Kirsch R: The diagnosis and management of Crohn's disease in populations with high risk rates for tuberculosis Aliment Pharmacol Ther 2007, 25:1373-1388.

[10]. Singh V, Kumar P, Kamal J,Prakash V,Vaipheik, Singh K: clinico colonoscopic profile of colonic tuberculosis .Am J Gastroenterol 1996:91:565_568.

[11]. Marschall JB: Tuberculosis of the gastrointestinal tract and peritoneum.Am J Gastroenterology 1993, 88: 989-999.

[12]. Had-Bojan Marjanovic ET alAbdominal localisation of Tuberculosis and role of surgery Medicine \& Biology Vol: 15 No 2, 2008 pp 51-55.

[13]. Antonis Michalopoulous et al Ceacal Obstruction due to primary intestinal tuberculosis: a case series Journal of Medical care Reports 2011: 5:128.

[14]. Rita S.Diagnosis of Abdominal Tuberculosis .Role of imaging J Ind Aced ClinMed 2001 July-Sept :2(3):103-04.

[15]. Kawaba FN. Abdominal Tuberculosis .A study of 881 cases JR Coll Surg Edin 1993:38(5):293. 